OPEN ACCESS

Edited by:

Alberto Porta,

University of Milan, Italy

Reviewed by:

John M. Karemaker,

University Medical Center Amsterdam, Netherlands

Roberto Maestri,

IRCCS Istituti Clinici Scientifici

Maugeri (ICS Maugeri), Italy

Antonio Roberto Zamunér,

Catholic University of the Maule, Chile

*Correspondence:

Darius A. Gerlach

Darius.Gerlach@dlr.de

${ }^{\dagger}$ These authors have contributed equally to this work as senior authors

Specialty section: This article was submitted to Autonomic Neuroscience,

a section of the journal

Frontiers in Neuroscience

Received: 13 December 2018

Accepted: 19 February 2019

Published: 05 March 2019

Citation:

Gerlach DA, Manuel J, Hoff A, Kronsbein H, Hoffmann F, Heusser K, Ehmke H, Diedrich A, Jordan J, Tank J and Beissner F (2019) Novel

Approach to Elucidate Human

Baroreflex Regulation at the Brainstem Level:

Pharmacological Testing During fMRI.

Front. Neurosci. 13:193.

doi: 10.3389/fnins.2019.00193

\section{Novel Approach to Elucidate Human Baroreflex Regulation at the Brainstem Level: Pharmacological Testing During fMRI}

\author{
Darius A. Gerlach ${ }^{1 *}$, Jorge Manuel' ${ }^{2}$, Alex Hoff', Hendrik Kronsbein ${ }^{1,3}$, \\ Fabian Hoffmann ${ }^{1,4}$, Karsten Heusser ${ }^{1}$, Heimo Ehmke ${ }^{3}$, André Diedrich ${ }^{5}$, Jens Jordan ${ }^{6}$, \\ Jens Tank ${ }^{1 \dagger}$ and Florian Beissner ${ }^{2 \dagger}$
}

\begin{abstract}
${ }^{1}$ Department of Cardiovascular Aerospace Medicine, Institute of Aerospace Medicine, German Aerospace Center (DLR), Cologne, Germany, ${ }^{2}$ Somatosensory and Autonomic Therapy Research, Institute for Neuroradiology, Hannover Medical School, Hanover, Germany, ${ }^{3}$ Institute of Cellular and Integrative Physiology, University Medical Center Hamburg-Eppendorf, Hamburg, Germany, ${ }^{4}$ Division of Cardiology, Angiology and Pneumology, Cologne Heart Center, University Hospital Cologne, Cologne, Germany, ${ }^{5}$ Division of Clinical Pharmacology, Department of Medicine, Autonomic Dysfunction Service, Vanderbilt University, Nashville, TN, United States, ${ }^{6}$ Chair of Aerospace Medicine, Institute of Aerospace Medicine, German Aerospace Center (DLR), Helmholtz Association of German Research Centers, Cologne, Germany
\end{abstract}

Introduction: Brainstem nuclei govern the arterial baroreflex, which is crucial for heart rate and blood pressure control. Yet, brainstem function is difficult to explore in living humans and is therefore mostly studied using animal models or postmortem human anatomy studies. We developed a methodology to identify brainstem nuclei involved in baroreflex cardiovascular control in humans by combining pharmacological baroreflex testing with functional magnetic resonance imaging.

Materials and Methods: In 11 healthy men, we applied eight repeated intravenous phenylephrine bolus doses of 25 and $75 \mu \mathrm{g}$ followed by a saline flush using a remote-controlled injector during multiband functional magnetic resonance imaging (fMRI) acquisition of the whole brain including the brainstem. Continuous finger arterial blood pressure, respiration, and electrocardiogram (ECG) were monitored. $\mathrm{fMRI}$ data were preprocessed with a brainstem-specific pipeline and analyzed with a general linear model (GLM) to identify brainstem nuclei involved in central integration of the baroreceptor input.

Results: Phenylephrine elicited a pressor response followed by a baroreflex-mediated lengthening of the RR interval (25 $\mu \mathrm{g}$ : $197 \pm 15 \mathrm{~ms} ; 75 \mu \mathrm{g}: 221 \pm 33 \mathrm{~ms})$. By combining $\mathrm{fMRI}$ responses during both phenylephrine doses, we identified significant signal changes in the nucleus tractus solitarii $(t=5.97)$, caudal ventrolateral medulla $(t=4.59)$, rostral ventrolateral medulla $(t=7.11)$, nucleus ambiguus $(t=5.6)$, nucleus raphe obscurus $(t=6.45)$, and several other brainstem nuclei $[p<0.0005$ family-wise error (few)-corr.]. 


\begin{abstract}
Conclusion: Pharmacological baroreflex testing during $\mathrm{fMRI}$ allows characterizing central baroreflex regulation at the level of the brainstem in humans. Baroreflex-mediated activation and deactivation patterns are consistent with previous investigations in animal models. The methodology has the potential to elucidate human physiology and mechanisms of autonomic cardiovascular disease.
\end{abstract}

Keywords: baroreflex, fMRI, brainstem, blood pressure, regulation, cardiovascular, nuclei

\section{INTRODUCTION}

Cardiovascular control centers in the brainstem govern arterial baroreflexes, which are important for human blood pressure buffering (Jordan et al., 2002) and long-term blood pressure control (Bisognano et al., 2011). Careful physiological investigations in animals showed that the NTS is the primary relay station for afferent input from carotid and aortic baroreceptors (McAllen and Spyer, 1976; Dampney, 1994). Connections from there to the NA and DMN elicit counter regulatory adjustment in efferent cardiac vagal activity. Projections to the cVLM and from there to the rVLM adjust efferent sympathetic traffic (Dampney, 1994; Dampney et al., 2002). Damage to these brainstem nuclei results in profound abnormalities in human blood pressure control. A patient with ischemic lesions involving bilateral NTS featured afferent baroreflex failure (Biaggioni et al., 1994). Degeneration of brainstem nuclei including the rVLM in patients with multiple system atrophy is associated with severe orthostatic hypotension among other disabling symptoms of efferent baroreflex dysfunction (Benarroch et al., 1998). Even subtle abnormalities in the structure or function of these nuclei could substantially affect human cardiovascular regulation. Yet, while the overall integrity of arterial baroreflex function can be interrogated with physiological and pharmacological baroreflex tests (Bristow et al., 1971, 1974), baroreflex regulation at the level of the brainstem is very difficult to measure in humans. Our goal was to develop a novel approach to assess human baroreflex regulation at the level of the brainstem. Therefore, we combined fMRI of the BOLD, beat-by-beat blood pressure and heart rate monitoring, and phenylephrine bolus injections for pharmacological baroreflex loading. Phenylephrine increases blood pressure leading to baroreflex-mediated vagal activation and sympathoinhibition. Brainstem fMRI has previously been validated for several applications including trigeminal pain research (Schulte et al., 2016), resting state connectivity measurement (Beissner et al.,

Abbreviations: $12 \mathrm{~N}$, hypoglossal nucleus; ANTs, advanced normalization tools; BOLD, blood oxygenation level dependent contrast; cVLM, caudal ventrolateral medulla; DMN, dorsal motor nucleus of the vagus nerve; DPGi, dorsal paragigantocellular nucleus; ECG, electrocardiogram; EPI, echo planar imaging; FOV, field of view; fMRI, functional magnetic resonance imaging; FSL, FMRIB Software Library; FWR-corr., family-wise error correction; GLM, general linear model; IO, inferior olivary nucleus; IRt, intermediate reticular nucleus; LBNP,lower body negative pressure; LPGi, lateral paragigantocellular nucleus; LRt, lateral reticular nucleus; MNI, Montreal Neurological Institute standard space; MPRAGE, 3D magnetization prepared rapid acquisition gradient echo; MRI, magnetic resonance imaging; NA, nucleus ambiguus; NTS, nucleus tractus solitarii; ROb, nucleus raphe obscurus; rVLM, rostral ventrolateral medulla; SBP, systolic blood pressure; SP5, spinal trigeminal nucleus; SpO2, oxygen saturation; SpVe, spinal vestibular nucleus; TE, echo time; TI, inversion time; TR, repetition time.
2014), characterization of the autonomic nervous system (Macefield and Henderson, 2010; Coulson et al., 2015), and studies on sleep apnea (Henderson et al., 2016).

\section{MATERIALS AND METHODS}

\section{Subjects}

We included 11 healthy, normotensive (125.7 \pm 4.6/73.4 $\pm 5.5 \mathrm{mmHg}, 57.6 \pm 7.5 \mathrm{bpm}$ ), non-smoking, men aged $30.5 \pm 6.3$ years with a weight of $78.0 \pm 10.6 \mathrm{~kg}$ ranging from 65 to $98 \mathrm{~kg}$ and a body mass index of $24.0 \pm 1.9 \mathrm{~kg} / \mathrm{m}^{2}$. Subjects were normally active and non-sedentary. The study complied with the Declaration of Helsinki was approved by the ethics committee of the Ärztekammer Nordrhein, Düsseldorf, Germany, and all subjects had given their written informed consent before inclusion. We registered the study under clinical trials registration number DRKS00013101 prior to commencement.

\section{Study Design}

The study was a randomized controlled interventional trial in an ambulatory setting. Subjects visited the facility on three different days. On the first study day, subjects were familiarized with the MRI environment and physiological recording equipment and underwent medical examination. On the second study day, the subject's baroreflex response to phenylephrine boli was examined. Finally, pharmacological baroreflex testing during fMRI took place on the third study day. Subjects rested before the scan and had abstained from caffeine and alcohol for $24 \mathrm{~h}$. After positioning and instrumentation of the subject followed by a resting period of $20 \mathrm{~min}$ in the scanner, we applied repeated intravenous phenylephrine (25 and $75 \mu \mathrm{g}, n=8$ ) boli followed by a $10 \mathrm{ml}$ normal saline flush using a programmable, MR-compatible remote-controlled injector. We repeated bolus administration every $120 \mathrm{~s}$ with $n=8$ boli in total. Each fMRI run lasted $16 \min 49 \mathrm{~s}$. The 25 and $75 \mu \mathrm{g}$ doses were applied in separate runs in randomized order. MRI measurements were carried out between 8:40 a.m. to 12:05 p.m. in an air conditioned room kept at a constant $21^{\circ} \mathrm{C}$. All subjects were asked about their mood, sleep, and mental state using a customized nonstandardized questionnaire before and after the examination. In particular, we asked about sleepiness, pain, and whether the subject perceived any effects of the injection.

\section{MRI Acquisition}

We obtained MRI acquisitions with a $3 \mathrm{~T}$ scanner (mMR Biograph PET-MRI scanner based on the Verio system, Siemens, 
Erlangen, Germany) with a 32-channel head coil. T1-weighted images for anatomical references were acquired using a MPRAGE sequence with the following parameters: TR: $2400 \mathrm{~ms}$, TE: $2.13 \mathrm{~ms}$, TI: $1000 \mathrm{~ms}$, flip angle: $8^{\circ}$, FOV: $246 \mathrm{~mm} * 192 \mathrm{~mm}$, matrix: $246 * 192$, slice thickness: $1 \mathrm{~mm}$. T2*-weighted functional images were acquired with an EPI sequence accelerated by multiband acquisition (TR/TE: $1180 / 32 \mathrm{~ms}$, flip angle: $64^{\circ}$, FOV: $180 \mathrm{~mm} * 208 \mathrm{~mm}$, matrix: $90 * 104$, slices: 78 with $2.0 \mathrm{~mm}$ slice thickness, voxel size: $2.0 \mathrm{~mm}$ isotropic, multiband factor: 6, volumes: 846; Xu et al., 2013; Todd et al., 2016). Unaccelerated single EPI images (TR: $6127 \mathrm{~ms}$, flip angle: 90 $)$ and B0weighted spin echo EPI (TR/TE: 12,000/102.6 ms) with matched and $180^{\circ}$ rotated phase encoding direction were acquired for better gray-white contrast and distortion correction, respectively. The flip angle was chosen according to the Ernst angle for shortened TR. Scans covered the whole brain including the brainstem. The total scan time was 45-50 min per intervention and subject. Image orientation was parallel to the anteriorposterior commissure line for T1-weighted images, whereas functional MRI images were additionally tilted by $\sim 35^{\circ}$ to avoid signal drop-outs in areas of interest. The MRI protocol was optimized according to the findings and recommendations from the human connectome project (Ugurbil et al., 2013). Additional information on MR imaging sequences for the non-specialist can be found in Bitar et al. (2006).

\section{Physiological Recordings}

We recorded beat-to-beat finger blood pressure with a modified device based on a commercially available finger blood pressure monitor $\left(\mathrm{NOVA}^{\circledR}\right.$, FMS, Finapres Measurement Systems, Amsterdam, Netherlands). For MRI compatibility, the device was radio frequency shielded and the cuff to frontend distance was prolonged to increase the frontend's distance to the scanner. We also acquired the ECG and $\mathrm{SpO}_{2}$ (MR400, PHILIPS, Orlando, FL, United States) as well as respiratory rate and end-tidal $\mathrm{CO}_{2}$ (etCO $\mathrm{CO}_{2}$;VY 450C, Branford, CT, United States). Signals were collected after A/D conversion (WINDAQ, DATAQ, Akron, $\mathrm{OH}$, United States) with a sampling frequency of $500 \mathrm{~Hz}$. Data preprocessing included peak detection, outlier filtering, resampling to fMRI acquisition, and normalization. RR intervals, systolic and diastolic blood pressure values, and respiration rate for each heartbeat and breathing cycle were analyzed. Subjects were equipped with active noise canceling headphones during the fMRI scan (OptoACTIVE, Optoacustics Ltd., Mazor,

\section{$1^{\text {st }}$ level analysis}

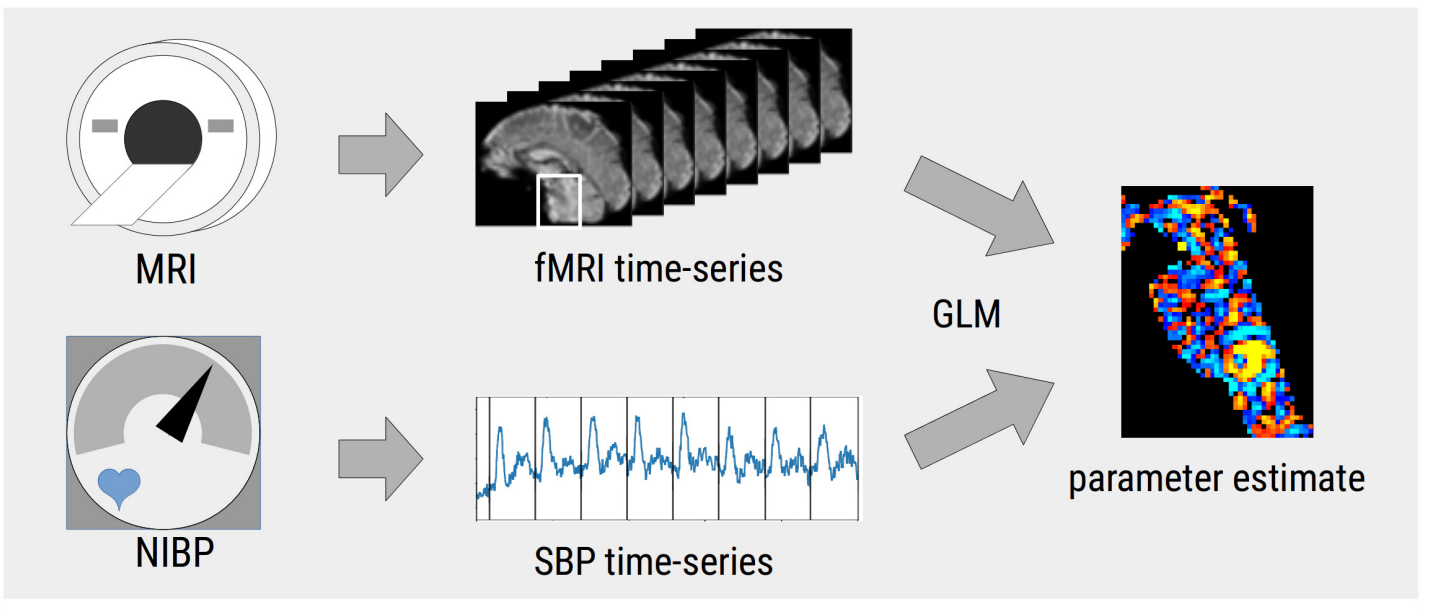

\section{$2^{\text {nd }}$ level analysis}

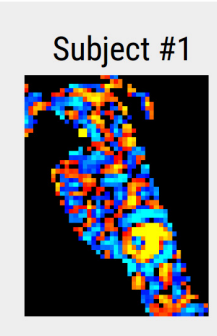

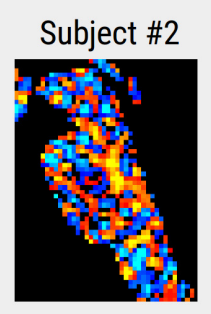

parameter estimates

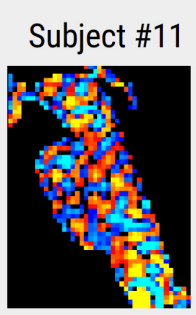

\footnotetext{
FIGURE 1 | Flow chart of the two-step statistical approach used in the study. In the first-level analysis, the time-course of systolic blood pressure (SBP) recorded during repeated phenylephrine bolus injections was used as regressor in a general linear model (GLM) on the functional MRI time-series leading to voxel-wise parameter estimate maps. In the second-level analysis, parameter estimates of all subjects were combined in a second GLM to calculate a group level statistical parametric map that was thresholded using family-wise error correction and threshold-free cluster enhancement. All analyses were restricted to the lower brainstem.
} 
Israel). A fixed head position was maintained by inflatable pads around the headphones.

\section{Image Analysis and Statistics}

We preprocessed fMRI data with FSL tools, v5.0.11 (Oxford Centre for Functional MRI of the Brain, Oxford, United Kingdom; Smith et al., 2004; Woolrich et al., 2009). After conversion of fMRI images to NIFTI format, all multiband EPI were realigned to the unaccelerated EPI image using FSL MCFLIRT. This approach allows for motion correction. At the same time, unwarping was conducted with FSL TOPUP using the spin-echo EPI for distortion correction (Andersson et al., 2003). Multiband EPI were then brain extracted with FSL BET, high-pass filtered with $180 \mathrm{~s}$ cut-off, and spatially normalized to a study template made from the T1 and unaccelerated EPI images of all subjects. Template and transformation for registration were calculated using ANTs (Tustison and Avants, 2013). The final preprocessing step was the upsampling to the T1 study template with ANTs.

The preprocessed data were cropped to retain only the lower brainstem and a brainstem mask was applied to remove adjacent areas with high physiological noise (Beissner et al., 2014). However, no spatial smoothing was applied in our study.

Statistical analysis was done with a mixed-effect GLM (Figure 1). The full SBP time-course of $16.5 \mathrm{~min}$ was used as explanatory variable and regressed against the BOLD signal time-courses of individual voxels. First-level (single-subject) analyses were performed with FSL_GLM and the parameter estimates passed up to a second-level (group) analysis using non-parametric permutation testing with FSL RANDOMISE. Significance was assumed at $p<0.0005$ corrected for multiple comparisons using family-wise error (FWE) correction and
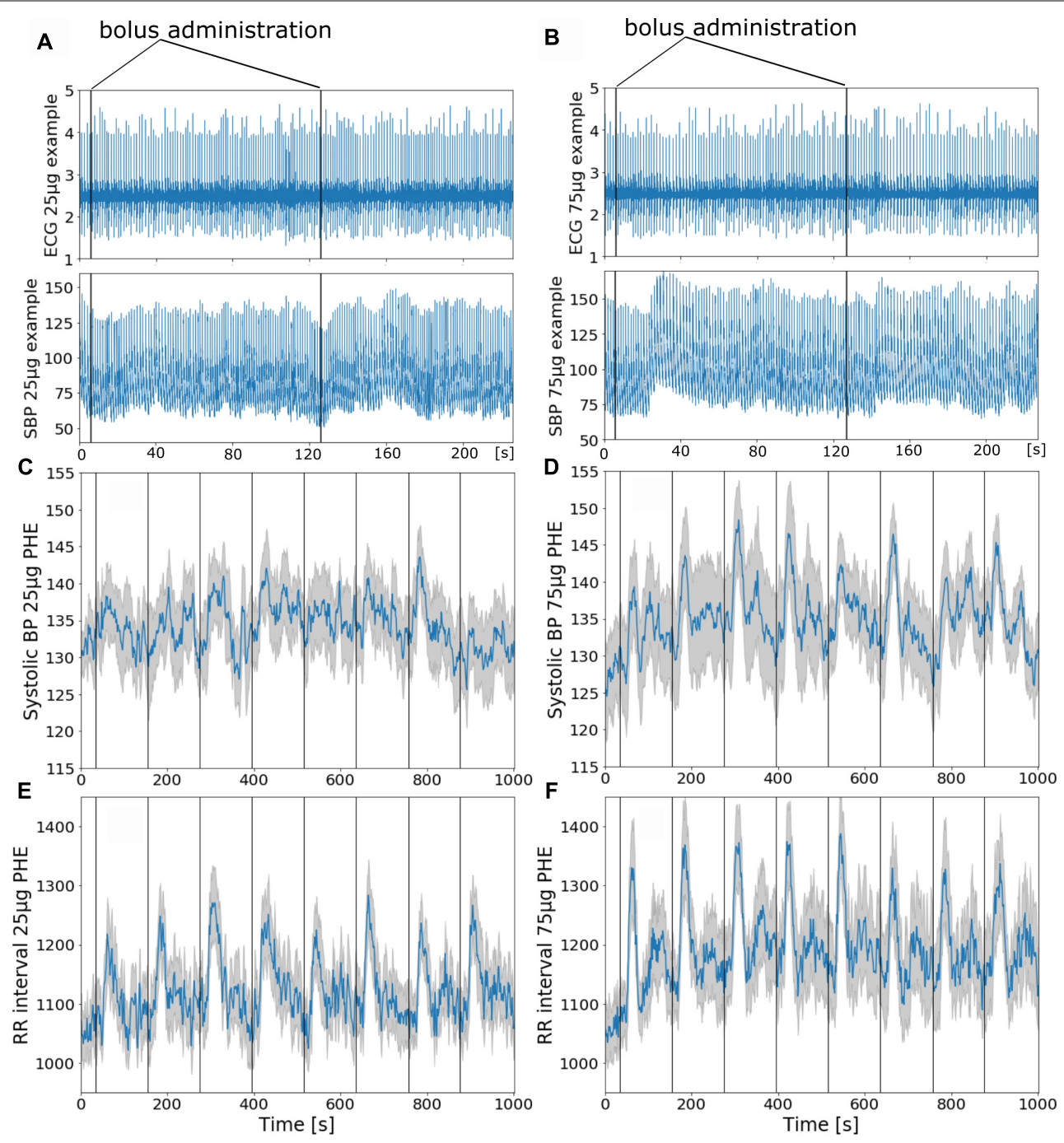

FIGURE 2 | (A,B) Phenylephrine bolus administration (A: $25 \mu \mathrm{g}$ and $\mathbf{B}$ : $75 \mu \mathrm{g}$ ) with blood pressure and ECG recordings during functional MRI. The vertical black lines indicate bolus injections. (C-F) Group mean \pm SE (gray curve) physiological recordings from 11 subjects with repeated phenylephrine administration. Systolic pressure (C) and RRI from ECG (E) recordings during $25 \mu \mathrm{g}$ phenylephrine and systolic pressure (D) and RRI (F) recordings during $75 \mu \mathrm{g}$ phenylephrine bolus administration. 
threshold-free cluster enhancement (Smith and Nichols, 2009). Results were reported in the form of $t$-values (defined as the parameter estimate from the GLM divided by the error of the parameter estimate). After statistical analysis, results were transformed to standard space (Montreal Neurological Institute, MNI152 $1 \mathrm{~mm}$ brain) using ANTs. We further analyzed the correlation between the averaged BOLD time-courses with the group mean SBP by extracting the BOLD signal from the masked lower brainstem.

\section{RESULTS}

\section{Pharmacological Baroreflex Testing}

All subjects reported to have slept well the night before the testing and were well motivated. None of the subjects reported pain provoked by the injection or by lying in the scanner, and all managed to stay awake throughout the test. We obtained good quality finger blood pressure recordings in most instances over the imaging period in 10 out of 11 subjects; in one subject, the signal drop outs were interpolated. Blood pressure traces deteriorated toward the end of the experiment in six of 11 subjects. To compensate for MRI induced artificial signal declines the regularly repeated level - and gain - calibration option (Physiocal ${ }^{\mathrm{TM}}$ ) was used in some subjects when needed. This resulted in a signal dropout of few seconds that was linearly interpolated. Still, all subjects could be considered for further analysis. Figures 2A,B illustrate continuous ECG and finger blood pressure recordings during administration of a $25 \mu \mathrm{g}$ and a $75 \mu \mathrm{g}$ phenylephrine bolus dose. Figures 2C-F illustrate averaged finger blood pressure RR interval responses to repeated phenylephrine doses; 25 and $75 \mu \mathrm{g}$ doses increased SBP $5 \pm 2$ and $15 \pm 2 \mathrm{mmHg}$, respectively. The pressor response elicited baroreflex-mediated heart rate reductions ( $R R$ interval lengthening $197 \pm 15 \mathrm{~ms}$ with $25 \mu \mathrm{g}$ : and $221 \pm 33 \mathrm{~ms}$ with $75 \mu \mathrm{g})$. Despite the relatively short period between the repeated boli of only $2 \mathrm{~min}$ blood pressure did not increase over the 16.5 min imaging time. Baroreflex sensitivity estimation resulted in mean values13.8 $\pm 7.4 \mathrm{~ms} / \mathrm{mmHg}$ for $25 \mu \mathrm{g}$ and $14.1 \pm 4.6 \mathrm{~ms} / \mathrm{mmHg}$ for $75 \mu \mathrm{g}$ ranging from 3.4 to $40.1 \mathrm{~ms} / \mathrm{mmHg}$. Baroreflex sensitivity was not always detectable especially during the $25 \mu \mathrm{g}$ doses.

\section{Brainstem fMRI}

The GLM of the BOLD signal with SBP revealed significant activations (i.e., positive correlation) on the group level. Thus, an increase in SBP was related to an increase of the BOLD signal in the respective voxels. The analysis was first conducted separately for 25 and $75 \mu \mathrm{g}$ phenylephrine doses, resulting in a single significant voxel for the $75 \mu \mathrm{g}$ runs that was located in the NTS. A paired $t$-test between the 25 and $75 \mu \mathrm{g}$ bolus administrations showed no differences, which led us to pool the data and improve statistical power. Based on this pooled analysis, we found significant activations in a number of brainstem nuclei that were subsequently identified using the Paxinos brainstem atlas (Paxinos et al., 2012). These nuclei comprised the NTS, cVLM and rVLM, ROb, DMN, nucleus hypoglossus (12N), inferior
TABLE 1 | Identified brainstem nuclei.

\begin{tabular}{|c|c|c|c|c|c|}
\hline Side & $t$-value & $\begin{array}{l}\text { MNI: } x \\
(\mathrm{~mm})\end{array}$ & $\begin{array}{l}\text { MNI: } y \\
(\mathrm{~mm})\end{array}$ & $\begin{array}{l}\text { MNI: } z \\
(\mathrm{~mm})\end{array}$ & Brainstem nuclei \\
\hline r & 7.21 & 5 & -38 & -60 & Inferior olive $(\mathrm{IO})$ \\
\hline \multirow[t]{3}{*}{1} & 7.11 & -8 & -38 & -46 & $\begin{array}{l}\text { Rostral ventrolateral medulla } \\
\text { (rVLM) }\end{array}$ \\
\hline & & & & & $\begin{array}{l}\text { Lateral reticular nucleus } \\
\text { (LRt) }\end{array}$ \\
\hline & & & & & $\begin{array}{l}\text { Lateral paragigantocellular } \\
\text { nucleus (LPGi) }\end{array}$ \\
\hline \multirow[t]{2}{*}{$1 / \mathrm{r}$} & 6.62 & 2 & -43 & -51 & Hypoglossal nucleus (12N) \\
\hline & 6.45 & 1 & -45 & -57 & $\begin{array}{l}\text { Raphe obscurus nucleus } \\
\text { (ROb) }\end{array}$ \\
\hline । & 6.29 & -3 & -43 & -56 & $\begin{array}{l}\text { Intermediate reticular } \\
\text { nucleus (IRt) }\end{array}$ \\
\hline \multirow[t]{2}{*}{ । } & 5.97 & -1 & -45 & -54 & $\begin{array}{l}\text { Dorsal motor nucleus of the } \\
\text { vagal nerve (DMN) }\end{array}$ \\
\hline & & & & & Nucleus tractus solitarii(NTS) \\
\hline r & 5.67 & 7 & -34 & -45 & $\begin{array}{l}\text { Rostral ventrolateral medulla } \\
\text { (rVLM) }\end{array}$ \\
\hline r & 5.67 & 2 & -40 & -44 & $\begin{array}{l}\text { Dorsal paragigantocellular } \\
\text { nucleus (DPGi) }\end{array}$ \\
\hline r & 5.61 & 7 & -42 & -56 & $\begin{array}{l}\text { Spinal trigeminal nucleus } \\
\text { (SP5) }\end{array}$ \\
\hline r & 5.6 & 6 & -40 & -48 & Nucleus ambiguus (NA) \\
\hline । & 4.98 & -7 & -44 & -56 & $\begin{array}{l}\text { Spinal trigeminal nucleus } \\
\text { (SP5) }\end{array}$ \\
\hline r & 4.59 & 6 & -38 & -55 & $\begin{array}{l}\text { Caudal ventrolateral medulla } \\
\text { (cVLM) }\end{array}$ \\
\hline । & 4.72 & -5 & -46 & -53 & $\begin{array}{l}\text { Spinal vestibular nucleus } \\
\text { (SpVe) }\end{array}$ \\
\hline I & 4.43 & -6 & -35 & -55 & Inferior olive $(\mathrm{IO})$ \\
\hline r & 4.4 & 6 & -45 & -50 & Medial vestibular nucleus \\
\hline । & 4.05 & -5 & -42 & -48 & $\begin{array}{l}\text { Nucleus tractus solitarii } \\
\text { (NTS) }\end{array}$ \\
\hline
\end{tabular}

All brainstem nuclei encompassed in the cluster were identified using a brainstem atlas. Active regions after $p<0.0005$ threshold are reported in MNI standard space coordinates with the corresponding t-values for the local maxima. (Larger $t$-values indicate better correlation between fMRI signal and systolic blood pressure. The $t$-value is defined as the parameter estimate from the GLM divided by the error of the parameter estimate.) Cluster size: $1437\left(\mathrm{~mm}^{3}\right)$, center of gravity $x: 1.46, y:-40$, z: -51.6. MNI: Montreal Neurological Institute.

olive (IO), and different reticular nuclei. MNI coordinates and corresponding $t$-values are shown in Table $\mathbf{1}$.

The relationship between the averaged time-courses of the whole lower brainstem BOLD signal and SBP is depicted in Figure 3. Both signals exhibit strong variations and a poor correlation $(R=0.25)$. Thus, blood pressure changes alone are most likely not the only contributor to the fluctuations of the BOLD signal.

Figure 4 illustrates the significant group level activations. Overlaid anatomical atlas slices were used to identify the nuclei.

\section{DISCUSSION}

The important finding of our study is that pharmacological baroreflex testing combined with fMRI reveals brainstem nuclei 


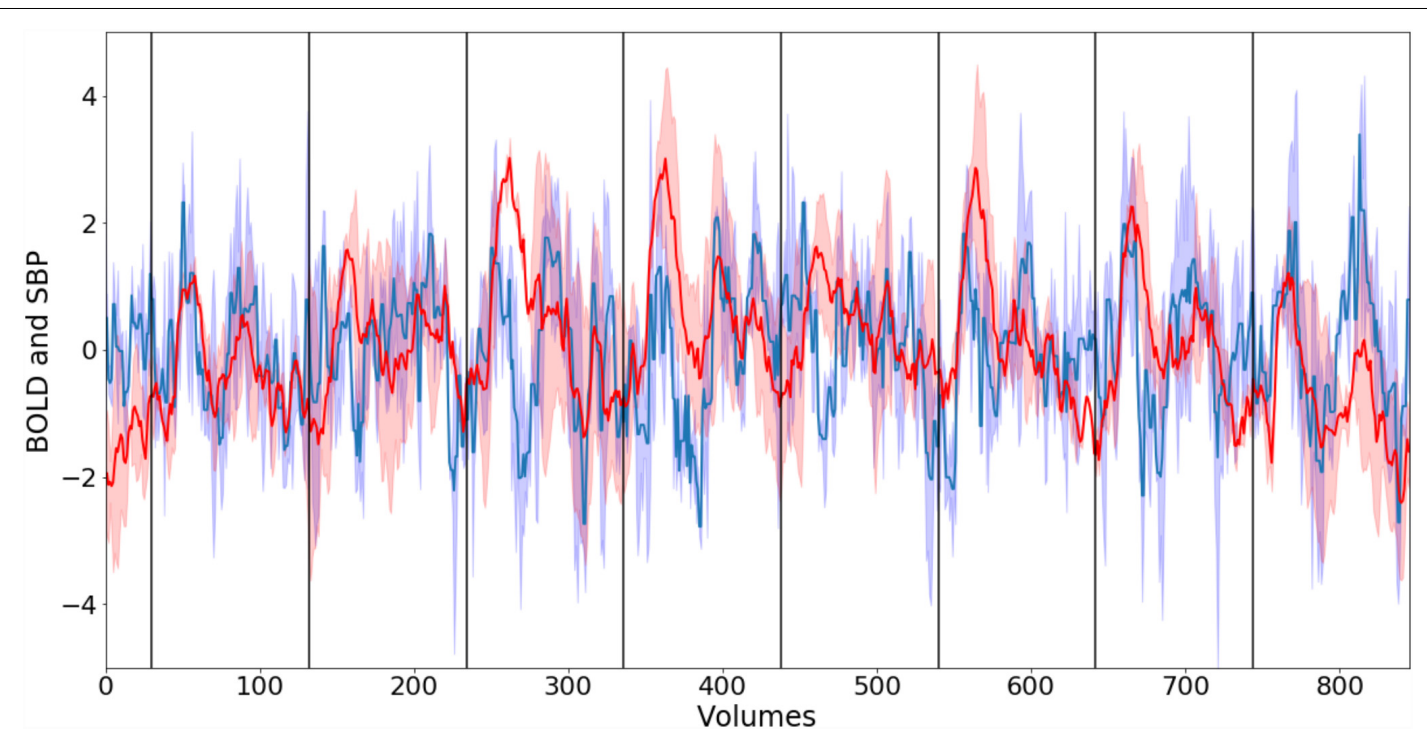

FIGURE 3 | Normalized smoothed group mean BOLD signal (blue) from lower brainstem and group mean systolic blood pressure (SBP) (red). Corresponding blue and red areas depict standard error. Black vertical lines indicate the start of phenylephrine bolus injections. The correlation $\mathrm{R}$ between both time-courses is 0.25 .

involved in human baroreflex regulation. In humans, we identified with high sensitivity all of the brainstem nuclei that have previously been shown to contribute to the baroreflex circuit in animals. Our approach can now be applied to elucidate the role of the human brainstem in cardiovascular physiology and in the pathogenesis of human cardiovascular disease.

The combination of continuous cardiovascular monitoring and brainstem fMRI during baroreflex loading with phenylephrine is a particular strength of our study. Beatby-beat blood pressure can so far only be assessed non-invasively with volume-clamp methods based on the Penàz servoplethysmomanometer (Molhoek et al., 1984). Devices based on this principle (i.e., NOVA ${ }^{\circledR}$, FMS, Finapres Measurement Systems, Amsterdam, Netherlands) are crucial for interrogating baroreflex function, need to be heavily modified for MRI studies and are only available in a few laboratories worldwide (Critchley et al., 2015). Other than the volume-clamp principle, there are commercially available non-invasive blood pressure systems for MRI (i.e., Biopac Systems Inc., Goleta, CA, United States) that are based on pulse decomposition analysis. The methodology is rather an indirect measure for the blood pressure (Baruch et al., 2011). Furthermore, brainstem fMRI is still challenging because of the strong physiological noise sources surrounding it (Beissner et al., 2014; Beissner, 2015). Due to the small size of brainstem nuclei, fMRI methods optimized for cortical structures cannot be applied (Beissner, 2015). Moreover, respiration, blood flow, and cerebrospinal fluid pulsations produce magnetic field distortions, out of phase spins and structural displacement (Brooks et al., 2013). Therefore, we aimed at maximizing statistical power by applying repeated stimulation with phenylephrine in a highly standardized fashion. To avoid non-specific effects of phenylephrine on brain circulation, we applied low and moderate phenylephrine doses (La Rovere et al., 1998). Baroreflex loading with phenylephrine produces an afferent signal that is conveyed to the NTS. Baroreflex afferent recordings in animal experiments and in patients during carotid surgery showed that signal time-course and magnitude were related to blood pressure. Since afferent nerve signals cannot be reasonably recorded in a human study, we utilized beat-by-beat blood pressure as input for our GLM analysis. We are aware that afferent baroreceptor input to NTS also feeds back on blood pressure. Compared with prior studies, our approach yields several advantages in delineating central baroreflex control. Previously applied autonomic challenges during fMRI include LBNP (Kimmerly et al., 2005), isometric handgrip testing (Coulson et al., 2015), Valsalva maneuver (Henderson et al., 2002), and slow breathing (Critchley et al., 2015). Handgrip and cold pressor testing engage central autonomic circuits through muscle afferents and pain fibers rather than baroreflex input. The Valsalva maneuver requires active participation likely confounding fMRI analysis and its effects are entangled with that of transient hypercapnia. The major challenge of LBNP is that it induces movement artifacts, when subjects are sucked down into the chamber. Moreover, the input stimulus for fMRI is commonly conceptualized as a boxcar time-course (on vs. off). Instead, we measured blood pressure time-course and magnitude during pharmacological baroreflex loading. It should be noted that BOLD contrast captures changes in neural activity but cannot readily differentiate neural inhibition and activation over time (Logothetis, 2008).

We reasoned that a methodology assessing brainstem baroreflex integration should recapitulate known baroreflex circuits. Indeed, baroreflex loading with phenylephrine yielded positive correlations between BOLD signals in the NTS and SBP. We also observed baroreflex-related BOLD signal changes in the NA which governs efferent cardiac vagal activity. Furthermore, baroreflex loading significantly changed BOLD signals in nuclei involved in sympathetic control including cVLM, rVLM, 


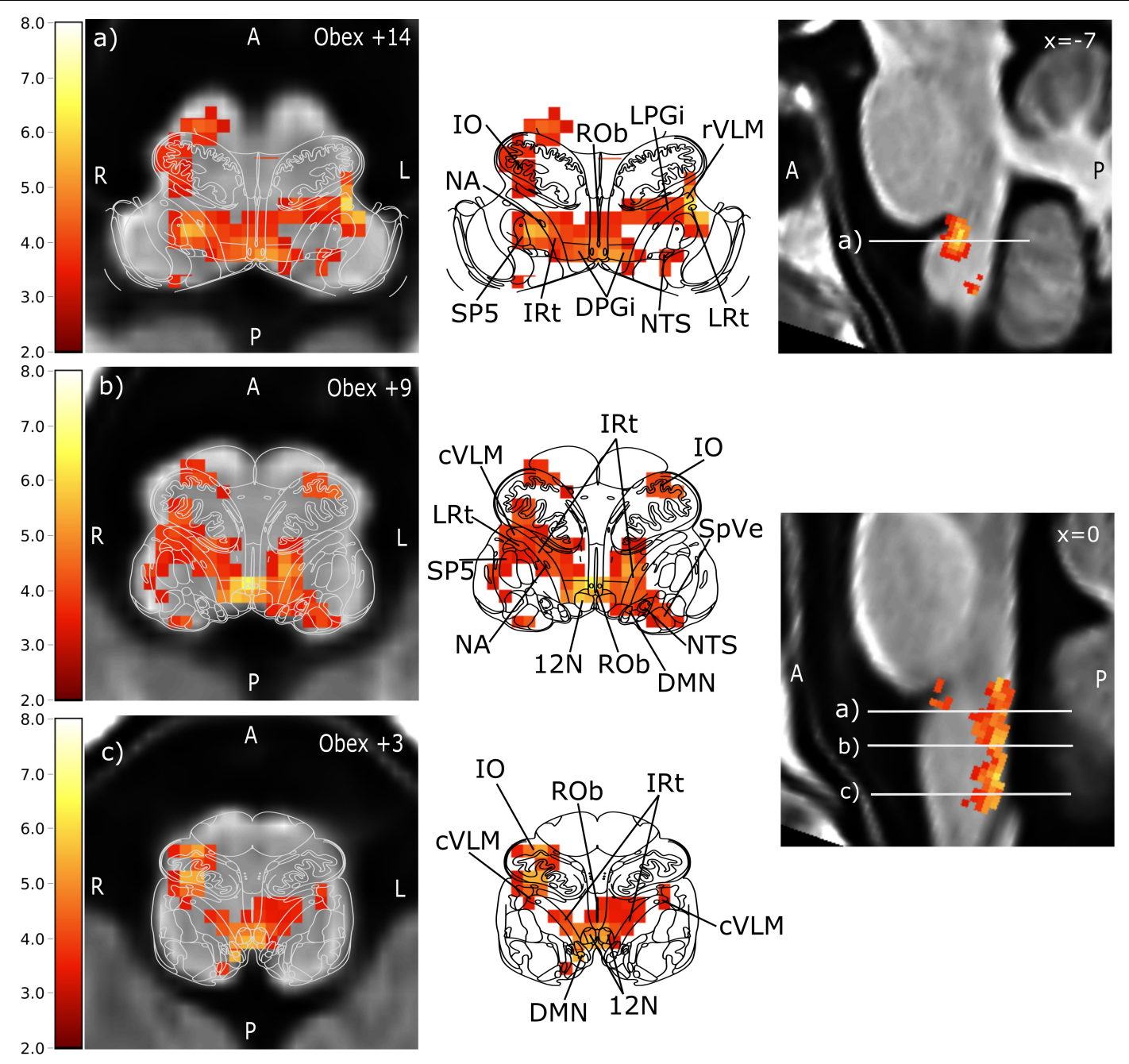

FIGURE 4 | Brainstem regions showing activations associated with blood pressure changes elicited by phenylephrine bolus injections. Group level results of all 11 subjects using data from all 16 boli. For visualization purposes, the images were tilted such that the resulting sections were perpendicular to the rostro-caudal brainstem axis to match the anatomical atlas. Left: transversal lower brainstem slices with of anatomical group template overlaid with the statistical parametric map of the positive BOLD correlation with SBP ( $t$-values encoded by color scale) and the corresponding atlas slice (modified from Paxinos brainstem atlas). Middle: BOLD overlay with the Paxinos brainstem atlas (Paxinos et al., 2012). Right: sagittal view of the brainstem. The corresponding transversal slices are marked by white lines with the letters of the sub-figure. Prominent activation maxima include (a) left rostral ventrolateral medulla (rVLM), Ncl. raphe obscurus (ROb), and right Ncl. ambiguus (NA), (b) Ncl. raphe obscurus (ROb), left intermediate reticular nucleus (IRt) extending to nucleus tractus solitarii, and right caudal ventrolateral medulla (cVLM), and (c) Ncl. hypoglossus (12N) extending to IRt and dorsal motor nucleus of the vagal nerve (DMN), right inferior olive extending to the cVLM. Further activated nuclei are: lateral reticular nucleus (LRt), dorsal paragigantocellular nucleus (DPGi), lateral paragigantocellular nucleus (LPGi), spinal trigeminal nucleus (SP5) and spinal vestibular nucleus (SpVe). A: anterior, P: posterior, L: left, R: right.

and raphe obscurus and provided more detailed coverage of baroreflex-regulated brainstem nuclei compared with previous fMRI studies in humans. Thus, fMRI resting state measurements combined with consecutively recorded muscle sympathetic nerve activity (Macefield and Henderson, 2010) suggested positive correlations between efferent sympathetic activity and the rVLM BOLD signal and negative correlations between sympathetic activity and NTS, cVLM BOLD signals. In another study, BOLD signals increased in broad regions including rVLM and decreased in cVLM and in medullary dorsomedial regions during inspiratory capacity apnea compared to relaxed breathing conditions (Macefield et al., 2006). Activation of higher cortical centers was reported in a baroreceptor unloading study with LBNP including insular frontoparietal cortex, and cerebellum (Kimmerly et al., 2005). Our localization of the rVLM, one of the central regions of baroreflex regulation, shows excellent correspondence with coordinates previously reported from a study correlating BOLD and blood pressure responses during hypoxia and normoxia and various breathing maneuvers (Critchley et al., 2015). Additionally, the IO, different reticular nuclei, $12 \mathrm{~N}$, and $\mathrm{ROb}$ were activated although they are traditionally not linked to blood pressure regulation. Even though some investigators suggested that these nuclei contribute to blood pressure control (Smith and Nathan, 1966; Miura and Reis, 1971), 
their role is not fully understood, yet. Because our methodology reliably identified previously known baroreflex-related brainstem nuclei, now human brain areas not previously accessible or not considered to relate to baroreflex activity can be interrogated.

\section{Limitations}

The BOLD signal is altered with changes in cerebral blood volume and blood flow (Logothetis, 2008). We cannot fully exclude that phenylephrine indirectly or directly affected cerebral circulation, which could affect fMRI analysis. In rabbits, phenylephrine increased blood pressure and cerebral blood flow while cerebral blood volume and de- and oxyhemoglobin were left unchanged (Koyama et al., 1990). The finding is reassuring since the latter influence the BOLD signal. Moreover, phenylephrine did not produce cerebral vasoconstriction in patients during anesthesia whereas the volatile anesthetic isoflurane increased cerebral blood flow (Strebel et al., 1998). In contrast, cerebral tissue oxygenation decreased with phenylephrine during anesthesia patients with unchanged cerebrovascular volume (Meng et al., 2012). However, none of these responses could explain specific BOLD signal changes in brainstem baroreflex circuits. Indeed, phenylephrine was also applied to elucidate baroreflex-mediated BOLD signal responses in cats before and after baroreceptor denervation (Henderson et al., 2004).

The number of subjects in our study is relatively low in comparison to conventional cortical fMRI studies, even though we were able to demonstrate significant activations based on the high number of stimuli used. Thus, our sample may not represent the average population, which should be considered when generalizing our results. However, most of our results are consistent with previous studies applying LBNP, Valsalva maneuver, hand grip, or inspiratory load (Macey et al., 2015). Finally, our spatial resolution of $2 \mathrm{~mm}$ isotropic limits our ability to clearly separate nuclei that are in close vicinity. For example, we cannot rule out that the activation of the DMN observed in our study was not based on signal spreading from the neighboring NTS. Future technological developments may mitigate this problem as may improved experimental designs.

\section{Perspectives}

We developed a novel approach to elucidate human baroreflex regulation at the level of the brainstem. The methodology can be applied to investigate human physiology. Indeed, much of our knowledge on central nervous baroreflex integration relies on animal studies and it has been difficult translating these findings to human subjects. Furthermore, the methodology can now be applied to investigate conditions associated with altered baroreflex function, dissect out the localization of the dysfunction, and, perhaps, target treatments in a more rational fashion. For example, fMRI-based baroreflex testing could be utilized to differentiate rare central and peripheral autonomic failure syndromes at an earlier stage. In common cardiovascular disorders such as heart failure, impaired baroreflex function heralds a poor prognosis (La Rovere et al., 1998, 2013). Better mechanistic understanding may beget new treatment approaches. Finally, device-based therapies targeting baroreflex afferents through electrical carotid sinus stimulation have been recently developed and tested in patients with resistant arterial hypertension and with heart failure (Heusser et al., 2010; Scheffers et al., 2010; Gronda et al., 2014). However, the response to electrical carotid sinus stimulation is variable and the proportion of non-responders is unacceptably high. Perhaps, brainstem studies could be developed further and then utilized to identify patients that are more or less likely to respond. To achieve these goals, the methodology should be tested in more detail, particularly in conditions associated with baroreflex impairment. Furthermore, the imaging methodology should be further refined. For example, more sophisticated analyses like masked independent component analysis (Moher Alsady et al., 2016) and frequency-based analysis of BOLD signals in restingstate settings (Chang and Glover, 2010) could prove useful.

\section{DATA AVAILABILITY}

The raw data supporting the conclusions of this manuscript will be made available by the authors, without undue reservation, to any qualified researcher. The datasets generated for this study are available on request to the corresponding author.

\section{AUTHOR CONTRIBUTIONS}

DG was the study coordinator and contributed to fMRI physiological data acquisition and analysis and writing of the manuscript. JM contributed to scripting of preprocessing, discussion of analysis and results, and revision of the manuscript. $\mathrm{AH}$ contributed to adjustment of fMRI compatible setup and physiological data acquisition. HK contributed to subject recruitment and physiological data acquisition. FH contributed to subject supervision and physiological data acquisition. $\mathrm{KH}$ contributed to data review and interpretation and revision of the manuscript. HE revised the manuscript. AD contributed to physiological data analysis. JJ contributed to data discussion and revision of the manuscript and was the supervisor. JT contributed to study idea and revision of the manuscript and was the project supervisor. FB contributed to study idea, statistical analysis, discussion of analysis and results, and revision of the manuscript.

\section{FUNDING}

This study was funded by the DLR internal cost object 2475023. FH was funded by University Hospital Cologne grant: 50WB1816 and DLR grant: 50WB1517.

\section{ACKNOWLEDGMENTS}

We are grateful for receiving the sequence "multibanded slice accelerated EPI with controlled aliasing" from the University of Minnesota Center for Magnetic Resonance Research. Furthermore, we are grateful to Finapres Measurement Systems (FMS, Amsterdam, Netherlands) for the extensive cooperation permitting customization of the finger blood pressure device NOVA ${ }^{\circledR}$ for MRI compatibility. 


\section{REFERENCES}

Andersson, J. L. R., Skare, S., and Ashburner, J. (2003). How to correct susceptibility distortions in spin-echo echo-planar images: application to diffusion tensor imaging. Neuroimage 20, 870-888. doi: 10.1016/S1053-8119(03)00336-7

Baruch, M. C., Warburton, D. E., Bredin, S. S., Cote, A., Gerdt, D. W., and Adkins, C. M. (2011). Pulse Decomposition Analysis of the digital arterial pulse during hemorrhage simulation. Nonlinear Biomed. Phys. 5:1. doi: 10.1186/17534631-5-1

Beissner, F. (2015). Functional MRI of the brainstem: common problems and their solutions. Clin. Neuroradiol. 25(Suppl. 2), 251-257. doi: 10.1007/s00062-0150404-0

Beissner, F., Schumann, A., Brunn, F., Eisentrager, D., and Bar, K. J. (2014). Advances in functional magnetic resonance imaging of the human brainstem. Neuroimage 86, 91-98. doi: 10.1016/j.neuroimage.2013.07.081

Benarroch, E. E., Smithson, I. L., Low, P. A., and Parisi, J. E. (1998). Depletion of catecholaminergic neurons of the rostral ventrolateral medulla in multiple systems atrophy with autonomic failure. Ann. Neurol. 43, 156-163. doi: 10. 1002/ana.410430205

Biaggioni, I., Whetsell, W. O., Jobe, J., and Nadeau, J. H. (1994). Baroreflex failure in a patient with central nervous system lesions involving the nucleus tractus solitarii. Hypertension 23, 491-495. doi: 10.1161/01.HYP.23.4.491

Bisognano, J. D., Bakris, G., Nadim, M. K., Sanchez, L., Kroon, A. A., Schafer, J., et al. (2011). Baroreflex activation therapy lowers blood pressure in patients with resistant hypertension: results from the double-blind, randomized, placebo-controlled rheos pivotal trial. J. Am. Coll. Cardiol. 58, 765-773. doi: 10.1016/j.jacc.2011.06.008

Bitar, R., Leung, G., Perng, R., Tadros, S., Moody, A. R., Sarrazin, J., et al. (2006). MR pulse sequences: what every radiologist wants to know but is afraid to ask. Radiographics 26, 513-537. doi: 10.1148/rg.262055063

Bristow, J. D., Brown, E. B., Cunningham, D. J. C., Goode, R. C., Howson, M. G., and Sleight, P. (1971). The effects of hypercapnia, hypoxia and ventilation on the baroreflex regulation of the pulse interval. J. Physiol. 216, 281-302. doi: 10.1113/jphysiol.1971.sp009525

Bristow, J. D., Brown, E. B., Cunningham, D. J. C., Howson, M. G., Lee, M. J. R., Pickering, T. G., et al. (1974). The effects of raising alveolarPCO2and ventilation separately and together on the sensitivity and setting of the baroreceptor cardiodepressor reflex in man. J. Physiol. 243, 401-425. doi: 10.1113/jphysiol. 1974.sp010760

Brooks, J. C., Faull, O. K., Pattinson, K. T., and Jenkinson, M. (2013). Physiological noise in brainstem FMRI. Front. Hum. Neurosci. 7:623. doi: 10.3389/fnhum. 2013.00623

Chang, C., and Glover, G. H. (2010). Time-frequency dynamics of resting-state brain connectivity measured with fMRI. Neuroimage 50, 81-98. doi: 10.1016/ j.neuroimage.2009.12.011

Coulson, J. M., Murphy, K., Harris, A. D., Fjodorova, M., Cockcroft, J. R., and Wise, R. G. (2015). Correlation between baseline blood pressure and the brainstem FMRI response to isometric forearm contraction in human volunteers: a pilot study. J. Hum. Hypertens. 29, 449-455. doi: 10.1038/jhh.2014.103

Critchley, H. D., Nicotra, A., Chiesa, P. A., Nagai, Y., Gray, M. A., Minati, L., et al. (2015). Slow breathing and hypoxic challenge: cardiorespiratory consequences and their central neural substrates. PLoS One 10:e0127082. doi: 10.1371/journal. pone. 0127082

Dampney, R. A. (1994). Functional organization of central pathways regulating the cardiovascular system. Physiol. Rev. 74, 323-364. doi: 10.1152/physrev.1994.74. 2.323

Dampney, R. A. L., Coleman, M. J., Fontes, M. A. P., Hirooka, Y., Horiuchi, J., Li, Y. W., et al. (2002). Central mechanisms underlying short- and long-term regulation of the cardiovascular system. Clin. Exp. Pharmacol. Physiol. 29, 261-268. doi: 10.1046/j.1440-1681.2002.03640.x

Gronda, E., Seravalle, G., Brambilla, G., Costantino, G., Casini, A., Alsheraei, A., et al. (2014). Chronic baroreflex activation effects on sympathetic nerve traffic, baroreflex function, and cardiac haemodynamics in heart failure: a proof-of-concept study. Eur. J. Heart Fail. 16, 977-983. doi: 10.1002/ ejhf.138

Henderson, L. A., Fatouleh, R. H., Lundblad, L. C., Mckenzie, D. K., and Macefield, V. G. (2016). Effects of 12 months continuous positive airway pressure on sympathetic activity related brainstem function and structure in obstructive sleep apnea. Front. Neurosci. 10:90. doi: 10.3389/fnins.2016.00090

Henderson, L. A., Macey, P. M., Macey, K. E., Frysinger, R. C., Woo, M. A., Harper, R. K., et al. (2002). Brain responses associated with the Valsalva maneuver revealed by functional magnetic resonance imaging. J. Neurophysiol. 88, 3477-3486. doi: 10.1152/jn.00107.2002

Henderson, L. A., Richard, C. A., Macey, P. M., Runquist, M. L., Yu, P. L., Galons, J. P., et al. (2004). Functional magnetic resonance signal changes in neural structures to baroreceptor reflex activation. J. Appl. Physiol. 96, 693-703. doi: 10.1152/japplphysiol.00852.2003

Heusser, K., Tank, J., Engeli, S., Diedrich, A., Menne, J., Eckert, S., et al. (2010). Carotid baroreceptor stimulation, sympathetic activity, baroreflex function, and blood pressure in hypertensive patients. Hypertension 55, 619-626. doi: 10.1161/HYPERTENSIONAHA.109.140665

Jordan, J., Tank, J., Shannon, J. R., Diedrich, A., Lipp, A., SchröDer, C., et al. (2002). Baroreflex buffering and susceptibility to vasoactive drugs. Circulation 105, 1459-1464. doi: 10.1161/01.CIR.0000012126.56352.FD

Kimmerly, D. S., O'leary, D. D., Menon, R. S., Gati, J. S., and Shoemaker, J. K. (2005). Cortical regions associated with autonomic cardiovascular regulation during lower body negative pressure in humans. J. Physiol. 569, 331-345. doi: 10.1113/jphysiol.2005.091637

Koyama, K., Mito, T., Takashima, S., and Suzuki, S. (1990). Effects of phenylephrine and dopamine on cerebral blood flow, blood volume, and oxygenation in young rabbits. Pediatr. Neurol. 6, 87-90. doi: 10.1016/0887-8994(90)90039-4

La Rovere, M. T., Bigger, J. T., Marcus, F. I., Mortara, A., and Schwartz, P. J. (1998). Baroreflex sensitivity and heart-rate variability in prediction of total cardiac mortality after myocardial infarction. Lancet 351, 478-484. doi: 10.1016/S01406736(97)11144-8

La Rovere, M. T., Pinna, G. D., Maestri, R., and Sleight, P. (2013). Clinical value of baroreflex sensitivity. Neth. Heart J. 21, 61-63. doi: 10.1007/s12471-012-0349-8

Logothetis, N. K. (2008). What we can do and what we cannot do with fMRI. Nature 453, 869-878. doi: 10.1038/nature06976

Macefield, V. G., Gandevia, S. C., and Henderson, L. A. (2006). Neural sites involved in the sustained increase in muscle sympathetic nerve activity induced by inspiratory capacity apnea: a fMRI study. J. Appl. Physiol. 100, 266-273. doi: 10.1152/japplphysiol.00588.2005

Macefield, V. G., and Henderson, L. A. (2010). Real-time imaging of the medullary circuitry involved in the generation of spontaneous muscle sympathetic nerve activity in awake subjects. Hum. Brain Mapp. 31, 539-549. doi: 10.1002/hbm. 20885

Macey, P. M., Ogren, J. A., Kumar, R., and Harper, R. M. (2015). Functional imaging of autonomic regulation: methods and key findings. Front. Neurosci. 9:513. doi: $10.3389 /$ fnins.2015.00513

McAllen, R. M., and Spyer, K. M. (1976). The location of cardiac vagal preganglionic motoneurones in the medulla of the cat. J. Physiol. 258, 187-204. doi: 10.1113/jphysiol.1976.sp011414

Meng, L., Gelb, A. W., Alexander, B. S., Cerussi, A. E., Tromberg, B. J., Yu, Z., et al. (2012). Impact of phenylephrine administration on cerebral tissue oxygen saturation and blood volume is modulated by carbon dioxide in anaesthetized patients. Br. J. Anaesth. 108, 815-822. doi: 10.1093/bja/aes023

Miura, M., and Reis, D. J. (1971). The paramedian reticular nucleus: a site of inhibitory interaction between projections from fastigial nucleus and carotid sinus nerve acting on blood pressure. J. Physiol. 216, 441-460. doi: 10.1113/ jphysiol.1971.sp009534

Moher Alsady, T., Blessing, E. M., and Beissner, F. (2016). MICA-A toolbox for masked independent component analysis of fMRI data. Hum. Brain Mapp. 37, 3544-3556. doi: 10.1002/hbm.23258

Molhoek, G. P., Wesseling, K. H., Settels, J. J., Van Vollenhoven, E., Weeda, H. W., De Wit, B., et al. (1984). Evaluation of the Penàz servo-plethysmo-manometer for the continuous, non-invasive measurement of finger blood pressure. Basic Res. Cardiol. 79, 598-609. doi: 10.1007/BF01910489

Paxinos, G., Huang, X., Sengul, G., and Watson, C. (2012). "Organization of brainstem nuclei. The human nervous system," in The Human Nervous System, eds J. Mai and G. Paxinos (Amsterdam: Elsevier Academic Press), 260-327. doi: 10.1016/B978-0-12-374236-0.10008-2

Scheffers, I. J., Kroon, A. A., Schmidli, J., Jordan, J., Tordoir, J. J., Mohaupt, M. G., et al. (2010). Novel baroreflex activation therapy in resistant hypertension: 
results of a European multi-center feasibility study. J. Am. Coll. Cardiol. 56, 1254-1258. doi: 10.1016/j.jacc.2010.03.089

Schulte, L. H., Sprenger, C., and May, A. (2016). Physiological brainstem mechanisms of trigeminal nociception: an fMRI study at 3T. Neuroimage 124, 518-525. doi: 10.1016/j.neuroimage.2015.09.023

Smith, O. A. Jr., and Nathan, M. A. (1966). Inhibition of the carotid sinus reflex by stimulation of the inferior olive. Science 154, 674-675. doi: 10.1126/science.154. 3749.674

Smith, S. M., Jenkinson, M., Woolrich, M. W., Beckmann, C. F., Behrens, T. E., Johansen-Berg, H., et al. (2004). Advances in functional and structural MR image analysis and implementation as FSL. Neuroimage 23(Suppl. 1), S208S219. doi: 10.1016/j.neuroimage.2004.07.051

Smith, S. M., and Nichols, T. E. (2009). Threshold-free cluster enhancement: addressing problems of smoothing, threshold dependence and localisation in cluster inference. Neuroimage 44, 83-98. doi: 10.1016/j.neuroimage.2008. 03.061

Strebel, S. P., Kindler, C., Bissonnette, B., Tschaler, G., and Deanovic, D. (1998). The impact of systemic vasoconstrictors on the cerebral circulation of anesthetized patients. Anesthesiology 89, 67-72. doi: 10.1097/00000542-19980700000012

Todd, N., Moeller, S., Auerbach, E. J., Yacoub, E., Flandin, G., and Weiskopf, N. (2016). Evaluation of 2D multiband EPI imaging for high-resolution, whole-brain, task-based fMRI studies at 3T: sensitivity and slice leakage artifacts. Neuroimage 124, 32-42. doi: 10.1016/j.neuroimage.2015. 08.056
Tustison, N. J., and Avants, B. B. (2013). Explicit B-spline regularization in diffeomorphic image registration. Front. Neuroinform. 7:39. doi: 10.3389/fninf. 2013.00039

Ugurbil, K., Xu, J., Auerbach, E. J., Moeller, S., Vu, A. T., Duarte-Carvajalino, J. M., et al. (2013). Pushing spatial and temporal resolution for functional and diffusion MRI in the human connectome project. Neuroimage 80, 80-104. doi: 10.1016/j.neuroimage.2013.05.012

Woolrich, M. W., Jbabdi, S., Patenaude, B., Chappell, M., Makni, S., Behrens, T., et al. (2009). Bayesian analysis of neuroimaging data in FSL. Neuroimage 45, S173-S186. doi: 10.1016/j.neuroimage.2008.10.055

Xu, J., Moeller, S., Auerbach, E. J., Strupp, J., Smith, S. M., Feinberg, D. A., et al. (2013). Evaluation of slice accelerations using multiband echo planar imaging at 3 T. Neuroimage 83, 991-1001. doi: 10.1016/j.neuroimage.2013.07.055

Conflict of Interest Statement: The authors declare that the research was conducted in the absence of any commercial or financial relationships that could be construed as a potential conflict of interest.

Copyright (๑) 2019 Gerlach, Manuel, Hoff, Kronsbein, Hoffmann, Heusser, Ehmke, Diedrich, Jordan, Tank and Beissner. This is an open-access article distributed under the terms of the Creative Commons Attribution License (CC BY). The use, distribution or reproduction in other forums is permitted, provided the original author(s) and the copyright owner(s) are credited and that the original publication in this journal is cited, in accordance with accepted academic practice. No use, distribution or reproduction is permitted which does not comply with these terms. 